\title{
A P-hub median network design problem with preventive reliability approach for before and after hub failure
}

\author{
ALIREZA EYDI ${ }^{1, *}$ and RAMIN NASIRI ${ }^{2}$ \\ ${ }^{1}$ Faculty of Engineering, University of Kurdistan, Pasdaran Blvd., Post Box no.: 416, Sanandaj, Iran \\ ${ }^{2}$ University of Kurdistan, Sanandaj, Iran \\ e-mail: Alireza.eydi@uok.ac.ir; ramin.nasiri1890@gmail.com
}

MS received 2 May 2018; revised 22 July 2018; accepted 29 August 2018; published online 9 February 2019

\begin{abstract}
Hubs are vital elements of communication and transportation networks and play an important role in interchanging the flows of information/passenger/goods. For this purpose, designing a highly reliable hub network is very critical, because inefficiency of even a single hub across the network tends to reduce the efficiency of the whole network in transferring the flow appropriately. In this research, a bi-objective mathematical model was designed to study the situations before and after hub failure. Considering reliability, the first objective was to maximize the flow through the network, and the second objective was to prevent wasting the flow due to a possible hub failure. The lexicographic method was used to solve this multi-objective problem with dependent objectives. This method represents an appropriate solution for problems whose objective functions are of different priorities or depend on one another. Various cases of different sizes were used to evaluate the model in terms of reliability. Since the hub location problem is an NP-Hard problem of commonly large dimensions, a hybrid meta-heuristic algorithm called "memetic algorithm" was used to have it solved. The algorithm was a combination of genetic algorithm with simulated annealing algorithm, where simulated annealing algorithm was used for local neighborhood search. Findings indicated that, consideration of the backup hub tends to enhance route reliability, thereby increasing the flow through the network, as compared to the case with no backup hub.
\end{abstract}

Keywords. Hybrid meta-heuristic algorithm; hub failure; reliability; backup hub; hub location.

\section{Introduction}

Hub location problem represents a new topic in the general field of location problems [1]. Hub is a general term referring to a location/point/place where goods or information from several sources come together before being dispatched to either other hubs along the network or the final destinations. A hub location problem determines location(s) of hub(s) and assigns network nodes to the hub(s), in such a way to minimize total costs for hub settlement and transportation. Hub location contributes into the movement of individuals, goods or information between an origin and a destination. As such, hub location problem seeks to optimally organize the movements and prevent unnecessary traffic, so as to bring about such advantages as lower fuel consumption, lower air pollution, lower equipment depreciation, and longer useful life of the network.

The decision on the locations of facilities is generally a long-term and strategic decision for large private and public companies. The large costs associated with such a decision necessitates system reliability assessment under various

*For correspondence conditions, so as to keep the system working at lowest possible cost even if an element along the network stops working for any reason. In a hub location problem, hub facilities are located in such a way to minimize all associated costs. On the other hand, in many cases, one or more of these facilities across the network may fail, making them unable to serve the system. These failures are of two major roots: (1) failure of the hub facilities, making them unable to serve the network, and/or (2) out-of-service communication routes. Thus, a highly important consideration in hub network design is the subject-matter of reliability. This research is aimed at presenting a mathematical model for P-hub median problem with preventive reliability approach for pre- and post-failure cases. By designing this model, we seek to not only maintain hub reliability but also prevent wasting the flow throughout the network as much as possible. A novelty of this research comes in the form of presenting a new and generalized model inspired from previous works on hub location problems along with novel formulations for real systems, where the reliability of communication routes is further considered. In this research, by creating and adding items of reliability and progressively correcting the model to maximize the flow 
throughout the network while allocating non-hub nodes to hub nodes, it is attempted to respond the demands optimally in a discrete space. All by all, the main purpose of this research is to develop a model for P-hub median problem where reliability concerns are considered. For this purpose, one should find optimum number of hubs and their locations for maximum reliability and preparedness for servicing along the communication routes. Accordingly, a biobjective mathematical model was designed. The first objective was to maximize the flow to enhance route reliability, and the second objective was to prevent wasting the flow due to hub failure by considering backup hub(s). Lexicography method was used to solve the problem. As the number of objectives increases and constraints emerge in practical systems, tuning the weighting parameters becomes an increasingly challenging stage. Chinchuluun and Pardalos indicated that lexicographic optimization is an ideal candidate for effective prioritization of multiple objective functions [2]. This method guarantees that improving the objectives of lower priority will not deteriorate the performance of higher-priority objectives. Given the dependence of the objective functions considered in this research, on one another, the lexicographic optimization approach was followed in this study. It was implemented in GAMS Software. Memetic hybrid metaheuristic algorithm (a combination of genetic and simulated annealing algorithms) has been used to solve problems of large dimensions [3]. Genetic algorithm is known to be susceptible to failure in local optimal traps. Therefore, here we combined the genetic algorithm with simulated annealing algorithm to achieve solutions of improved quality. Actually, this research follows a redundancy approach to enhance reliability by considering backup elements across the system. In the present research, backup hubs were used to either enhance route reliability (before a possible hub failure) or maximize flow capacity across the designed network (after a possible hub failure).

The remainder of this paper is organized as follows. Section 2 presents a review on related literature. The considered problem is explained in section 3, with the proposed mathematical model described. Sections 4 and 5 present the memetic algorithm and solution representation. Numerical results are provided and discussed in section 6 . The conclusions are drawn in section 7.

\section{Literature review}

The term hub refers to a collection and/or distribution center midway along one or more indirect paths, rather than having a direct relationship between two points. In a hub location problem, the objective is to find hubs and routes for dispatching flow from a series of origins to a series of destinations, in such a manner that the process of flow distribution and dispensation becomes optimum [4]. By flow, we mean individuals, goods, or information, depending on the scope of the problem in question. The final goal is to reduce associated expenses with each hub and each route and also to obtain the required financial profit via various trips between hubs. The flow is dispatched from the origin to the hubs where it is reorganized before being resent to either another hub/middle destination or the final destination. In general, the purpose of establishing a hub network is to provide an infrastructure on which the flow can be moved from the middle nodes at a lower cost.

The importance of hub is expressed when there is a need for "saving in scale" in the cost of trips or communications along particular routes. Saving in scale means that, in an air network, for example, one can use larger and more efficient airplanes along the lines connecting the hubs. When it comes to communication networks, the use of light fibers of broader capacity for establishing connections between pairs of hubs may represent the saving in scale. Indeed, total transportation cost per unit good between the hubs is lower than that from the origin to the hub and then from the hub to the destination; i.e., transportation via the hubs reduces total cost. Hub-based networks are composed of two parts: (1) the communication network between non-hub nodes and hubs, which is called tributary, and (2) hub-connecting network which is called backbone.

In these types of problems, locations of hub facilities are selected from a pool of nodes, with the remaining nodes allocated to different hubs. This allocation is performed in such a way that there is a route between each origin and each destination. In reality, hub location problem is a location-allocation problem which is widely applied in the field of location problems. In these types of problems, the goal is to minimize associated costs with establishing several new facilities among the existing facilities and optimal allocation of the newly established facilities to the existing facilities. Hub location problem can be observed as a location allocation problem where hubs are located firstly, followed by allocating non-hub nodes to the hub nodes. It is important to consider both location and allocation problems when designing a hub network because these tend to affect one another. That is, optimum hub allocation depends on hub location and the vice versa.

As mentioned in the previous section, hubs comprise a vital element of transportation systems. As such, there are chances that, for virtually any reason (e.g., weather condition, labors strike, sabotages, or even a change in system ownership), one or more hubs fail to serve the system appropriately. The failure of these facilities can end up with additional transportation costs as the customers are likely to travel longer distances in such circumstances. For this reason, it is highly necessary to account for reliability when locating a hub-based network. Following with this section, research works performed in this context will be briefly described.

Investigated in this section are the studies where facility location and allocation problems are explicitly considered 
by taking the subject-matter of reliability into account since 2003. Snyder's doctoral thesis was the first to study location-allocation problem considering the subject-matter of reliability. Following a new approach to supply chain optimization under uncertainty, Snyder and Daskin was the first to directly consider facility failure as facility reliability in the location-allocation problem [5]. He mathematically modeled two cases of location-allocation problems, namely Uncapacitated Facility Location Problem (UFLP) and P-Median Problem, considering the subject-matter of reliability for the facilities to be located. He performed the modeling following either a single-objective approach to minimize maximum failure cost or a bi-objective approach to minimize operational costs (per day transportation cost when all facilities are operating normally) and expected failure costs (expected transportation costs when facility failure occurs). He then used Lagrangian relaxation method to determine upper and lower bounds of the problem before adopting branch and bound method to obtain an optimum solution to the problem. He further developed a trade-off curve based on the cures of operational and expected costs and calculated expected failure costs for various operational costs, so as to illustrate that, system reliability can be enhanced remarkably by increasing the operational costs slightly. Berman et al investigated P-median problem considering reliability issues and demonstrated that, considering the asymptotic property of the problem, the P-median problem under uncertainty converges to the $\mathrm{P}$-median problem under certainty when failure chance is zero [6]. They also studied P-median problem under uncertainty under a worst-case scenario and presented heuristic methods of different upper and lower bounds for solving the problem. In his doctoral thesis, Zhan and Shen studied facility location problem with fixed prices both for single-level and multi-level cases considering unsteady (unreliable), facilities and presented a mixed-integer programming model for the problem [7]. He further presented efficient techniques based on genetic algorithm for solving the model. In addition, Zhan investigated reliability enhancement of a pre-designed and already installed system mathematically. Lim et al studied UFLP considering reliability and presented an integer mathematical model for the problem when the facility robustness was a concern [8]. They further solved the prepared model using Lagrangian relaxation. With such a large number of models and various designs proposed by different designers working on distribution, transportation, and remote communication networks, one may raise the expectation that reliability is already recognized as an essential element of such systems, because any defect (i.e., unreliability) can distort the network seriously and not only impose major impacts on the flow of providing services and revenue creation, but also incur large costs for the systems [9]. For instance, some of the facilities may become inactive as a result of natural disasters, labors strikes, or terroristic threats. Given that, once produced or processed, the materials or information flows shall be distributed through distribution facilities, any failure in the facilities will create major problems for the entire system, ending up with large economic losses (see ref. [10]). Hub location has been acknowledged as an important operational factor in remote communication networks. In order to prevent failures across these networks, the focus of preventive strategies is on the locations where hub facilities are deployed [11]. Given the increased vulnerability of hub facilities to possible failures, design of a reliable hub network represents a significant challenge in the present communication systems $[12,13]$. In a paper by Grubesic et al, a great deal of attention was paid to geographical and operational concentration of hubs, and the fact that companies see those vital to preserve high speed and wide bandwidth of their communication networks [11]. Even though geographical closeness among the hubs reduces the possibility of missing traffic for transferring total flow and brings about some other advantages by increasing the capacity of the hub activities, but there are chances that, due to a reduction in the network performance resulted from disorders, the activities are disordered and the system is endangered. In contrast, O'Kelly offered a hub location model called "reliable hub location" which was focused on maximizing network performance by finding and settling hubs at locations where the probability of distortion in inter-city flows was minimal, i.e., the probability for reaching the destination was maximum [14].

Research works on reliability can be classified into three general classes [5]: (1) network reliability, (2) consideration of redundancy or support, and (3) hybrid methods for increasing network reliability. In the previous section, we investigated the first approach by studying related research works. But the second and third approaches have been rarely discussed in the literature. Accordingly, the present study is focused on these two classes of the research works. Parvaresh et al presented the first and the most important research work on redundancy or support in hub location problems [15]. In this research, the researchers formulated a multi-allocation P-hub median location problem based on games theory where the hubs were exposed to previously designed attacks. Briefly, the model recognized unavailable hubs and considered a set of backup hubs to replace the unavailable hubs in such a way that the system ends up with least transfer cost. They also used two heuristic algorithms derived from simulated annealing method for solving their problem. Later on, Hamidi et al followed a figurative hub approach in their research to confront terrorist attacks [16]. In their approach, a simple single-allocation hub location problem with fake hubs and active routes was considered. Following this approach, system reliability could be increased before a possible defect may occur, while making it difficult to recognize main and fake hubs for the terrorist groups. Azizi et al incorporated backup hubs into a singleallocation hub location problem [17]. They began by considering all hypotheses of classic P-hub median location problem and then added to it hypothetical backup hubs 
which could be used when primary hubs were not accessible. Their goal was to minimize the transportation cost through the main hubs. They used a genetic meta-heuristic algorithm to solve the problem, so as to expand their previous research, Azizi et al presented a nonlinear mathematical model for the hub location problem where every demand point had a backup hub to serve the network in case the original hub failed [18]. Other works in this field include the research by Snyder et al [10] who, similar to Azizi et al [17], considered not only backup hubs, but also backup routes. They assumed possible failure of one or more hubs independently. For this purpose, they tried to consider a reliable substitute for each route/hub. The more important point they referred to, as compared to the work by Azizi et al [17], was that, not only selective hubs could be chosen as backup hubs, but also a non-hub node could be used as a backup hub. We may further refer to the work by Eghbali et al [19] who used a bi-objective hub location problem. They followed a coverage approach to network reliability enhancement. They also considered a minimum threshold limit for the network reliability, so that a transfer was possible only when this minimum network reliability was satisfied. Zarrinpoor et al proposed a novel reliable hierarchical location-allocation model. The model was able to account for the uncertainty associated with input parameters. They used an accelerated Benders decomposition algorithm to solve the model [20]. Ghezavati and Hosseinifar presented a bi-objective mathematical model for facility location problem where customer's demand was assumed to vary stochastically. The bi-objective function sought to (1) minimize the risk and (1) minimize the total cost of the network. They used $\varepsilon$-constraint, non-dominated sorting genetic algorithm-II (NSGA-II), and multi-objective particle swarm optimization (MOPSO) methods to solve the bi-objective model [21].

According to Sohn and Park [22], hub location problem is an NP-hard problem for which various solution methods have been proposed. Exact solution methods such as Benders decomposition, and branch-and-price technique were developed by Contreras et al [23] who used this algorithm for solving problems of small dimensions. O'Kelly presented a near-optimum method for a single-allocation hub location problem without imposing any constraint on capacity [24]. Rodriguez et al used a simulated annealing algorithm for solving the P-hub median problem with multiple allocations [4].

\subsection{Hybrid metaheuristic literature}

Recently, many research works have been performed on hybrid algorithms. In many cases, including real examples, the best solutions are obtained from these algorithms. Early research works were focused on combinations of more than one meta-heuristic algorithms; but later on, various combinations of meta-heuristic and exact algorithms were developed. Providing the advantages of both types of algorithms, these algorithms are proved to be very useful. The memetic algorithm (MA) is a well-known instance of such hybrid methodologies. In this scheme, the genetic algorithm can be replaced by, for example, local search algorithm using an operator which is usually mutation. For many combinatorial optimization tasks, it has been shown that it is essential to involve some improvement strategies into a heuristic method to yield effective optimization tools. MA is a metaheuristic method developed by incorporating local search heuristic into the traditional evolutionary concept. Acampora et al used a local search technique to direct the evolutionary algorithm to promising regions of the search space, although premature convergence to a local optimum has been always a potential threat [25]. MAs have shown large potentials for solving various problems. For fundamental, theoretical aspects of MAs and an overview on advanced, practical applications of the technique, refer to [26, 27]. Chen offered a hybrid heuristic method for solving the P-hub median problem with limited capacity. Their method provided optimum solution in small problems and its solution was still better than simulated annealing algorithm for large problems [28]. Pradeepmon and Paul offered a hybrid meta-heuristic algorithm for facility location problem with limited capacity [29]. Saboury et al proposed a combination of simulated annealing algorithm with nearest-neighbor search (NNS) for a fully connected bi-level network [30]. Particle swarm optimization-nearestneighbor search (PSO-NNS) hybrid method was proposed for hub location problem by Mokhtari and Abbasi [31]. As of now, no research has considered the reliability both before and after facility failure, and most of related research works on hub location problem with reliability have considered the reliability either before or after such a failure (rather than both of them at the same time).

AminNaseri et al presented a bi-objective mathematical model for a single hub location-allocation problem, which travel times were assumed to be of non-deterministic nature. The aim was to find a set of locations for potential hubs and assign them to non-hub nodes to minimize total transportation costs while maximizing the network uncertainty [32]. Zhalechian et al proposed a novel scenario-based model to design a resilient hub network and presented an objective function to calculate the hub network resilience. They further presented a hybrid evolutionary algorithm to solve the model for large-scale problems [33]. Moghal et al developed a mixed-integer linear programming model for minimizing transportation costs and inventory of food grains in India, and also used a chemical reaction optimization (CRO) algorithm for testing the model, ending up indicating superior computational performance of the model, as compared to similar meta-heuristic algorithms [34]. Kertal et al presented a hub network model where optimal hub location and routing was taken into account. They further considered simultaneous pick-up and delivery by vehicles to achieve higher efficiency. They proposed a hybrid heuristic of multi-start simulated 
annealing and ant colony system for solving the problem [35]. Bashiri et al developed a mathematical model for the Pmobile hub location problem to improve network efficiency and used a genetic algorithm augmented by a local search algorithm to solve large instances of the proposed model [36]. In another research, Mogale et al developed an integrated multi-objective, multi-modal and multi-period model for the grain silo location-allocation problem with dwell time. They further compared their results to those of NSGA-II algorithm [37]. Mogale et al formulated a Mixed-Integer Non-Linear Programming (MINLP) model for planning the food grain storage and movement from surplus states to deficit states considering the seasonal procurement, silo capacity, demand satisfaction, and vehicle capacity constraints. The formulated problem was solved by a Hybrid Particle-Chemical Reaction Optimization (HP-CRO) algorithm [38]. Maiyar and Thakkar formulated a combined tactical and operational deterministic two-stage food grain transportation model with linear and MINLP formulations in the first and second stages, respectively. Evaluating the model on a case study from India, they used PSOCP and PSO to solve the problem [39]. Mogale et al developed a MINLP model for bulk wheat transportation and storage problem via public distribution system and proposed Hybrid Chemical Reaction Optimization with Tabu Search (CROTS) for solving the problem [40].

\section{Problem description}

We have modeled our problem on the basis of the following assumptions:

- The problem under study is a median hub problem.

- The designed network is a full-hub one.

- The network transfer capacity is unlimited.

- Any hub failure may occur independently.

- Hubs are subject to single allocation.

- No more than one hub can be failed at the same time.

- Reliability of the transfer between each pair of hubs is indicated by a number in the interval $[0,1]$.

- Potential hub locations are specified and located in discrete space.

- If a hub goes out of service, no flow is permitted to pass through that hub. The flow is rerouted via one of the backup hubs in the network.

- Network reliability is calculated following a serial approach.

- This paper addresses hub location problem with probabilistic disruptions.

\subsection{Indices}

$i$ : $\quad$ Origin node

$n$ : Total number of network nodes

$j$ : Destination node $k$ : The first hub along the network

$m$ : The second hub along the network

$l$ : Backup hub

\subsection{Parameters of the problem}

$P: \quad$ Number of hubs to be located

$W_{i j}$ : $\quad$ Flow between the origin and destination nodes

$r_{i k m j}$ : Reliability of the flow between the origin and destination nodes

$q_{k}$ : $\quad$ Probability of the failure for the hub $k$

$\alpha$ : Reliability of the flow between two hub nodes (the higher the reliability of the flow between hubs, the higher the entire network reliability will be)

In a P-hub median problem, each node is allocated to one or more particular hubs, and all of the input and output flows are routed by that hub. The route from the origin $i$ to the destination $j$ consists of three segments:

1. Collecting the parcel from the origin toward the hub $k$

2. Transferring the parcel through the first hub $k$ toward the second hub $m$

3. Distributing the parcel from hub $m$ toward the destination $j$

The reliability of the route $i \rightarrow k \rightarrow m \rightarrow j$ is given by $r_{i k m j}=r_{i k} * r_{k m}^{(1-\alpha)} * r_{m j}$ which has been taken from O'Kelly [11].

\subsection{Decision variables}

$$
\begin{gathered}
\hline \begin{array}{c}
=1 \text { if non-hub node } i \text { is allocated to hub } k, \\
=0 \text { otherwise. }
\end{array} \\
\begin{array}{c}
x_{i k m j}=1 \text { if the connection between the origin } i \text { and the } \\
\text { destination } j \text { is established via hubs } k \text { and } m, \\
=0 \text { otherwise. } \\
=1 \text { if hub } k \text { is allocated to hub } l, \\
=0 \text { otherwise. }
\end{array}
\end{gathered}
$$

In figure 1, different types of the routes in a single-level hub network are shown. Each route is defined as a variable with its reliability specified.

In this section, the approach toward modeling the considered P-hub median location problem with reliability considered is described.

In the proposed model, total flow when all of the hubs function properly is referred to as reliability-based total flow, $r_{i k m j}$ and can be calculated from Eq. (1):

$$
T T W=\sum_{i} \sum_{k} \sum_{m} \sum_{j} r_{i k m j} x_{i k m j} w_{i j}
$$

Once finished with calculating the flow under normal conditions, the aim is to maximize the flow in case of a hub failure. For this purpose, when hub $k$ is failed and 


\begin{tabular}{|l|l|l|}
\hline Type of route & $\begin{array}{c}\text { Decision } \\
\text { variables }\end{array}$ & Route reliability \\
\hline & $x_{i k m j}$ & $r_{i k} r_{k m}^{(1-\alpha)} r_{m j}$ \\
\hline & $x_{k k m m}$ & $r_{i k} r_{k k}^{(1-\alpha)} r_{k j}$ \\
\hline & $x_{i k k k}$ & $r_{k k} r_{k m}^{(1-\alpha)} r_{m m}$ \\
\hline
\end{tabular}

Figure 1. Calculation of reliability across the network.

considering hub $l$ as the backup hub, the reliability-based flow can be obtained from Eq. (2):

$$
T R W B_{k l}=\sum_{i \neq k} \sum_{m \neq k} \sum_{j \neq k} r_{i l m j} w_{i j} x_{i k m j}+\sum_{i \neq k} \sum_{j \neq k} r_{i l l j} w_{i j} x_{i k k j}
$$

Equation (2) indicates re-routing in the two modes: (1) there are two hubs along the route, and (2) there is only one hub along the route. Equation (3) indicates the flow after rerouting when the second hub is failed:

$$
T R W B_{m l}=\sum_{i} \sum_{k \neq m} \sum_{j \neq m} r_{i k l j} w_{i j} x_{i k m j}
$$

\subsection{Mathematical formulation of the problem}

In this research, two objective functions were considered, as described in the following:

$$
\begin{gathered}
\max \operatorname{obj} 1=\sum_{i} \sum_{k} \sum_{m} \sum_{j} r_{i k m j} x_{i k m j} w_{i j} \\
\max o b j_{2}=\sum_{i} \sum_{k} \sum_{m} \sum_{j} r_{i k m j} x_{i k m j} w_{i j} \\
+\sum_{k} \sum_{l} T R W B_{k l} u_{k l} q_{k} \\
+\sum_{m} \sum_{l}^{l} T R W B_{m l} u_{m l} q_{m}
\end{gathered}
$$

According to Eq. (4), total flow is maximized when the hubs operate normally, i.e., at maximum reliability. According to Eq. (5), the first part sums up the flow when a hub fails and the flow when the hub is working normally, so as to maximize total flow. The second and third parts calculate the flow when the hubs $k$ and $m$ are failed, respectively. The following constraints were imposed on the model:

$$
\begin{gathered}
\sum_{k} z_{k k}=p \\
\sum_{k} z_{i k}=1 \quad \forall i \\
\sum_{m} x_{i k m j}=z_{i k} \quad \forall i, j, k \\
\sum_{k} x_{i k m j}=z_{i k} \quad \forall i, j, m \\
\sum_{l \neq k} u_{k l}=z_{k k} \quad \forall k \\
u_{k l} \leq z_{k k} \quad \forall k, l \neq k \neq l
\end{gathered}
$$$$
T R W B_{k l}=\sum_{l \neq k} \sum_{m \neq k} \sum_{j \neq k} r_{i l m j} w_{i j} x_{i k m j}+\sum_{i \neq k} \sum_{j \neq k} r_{i l l j} w_{i j} x_{i k k j}
$$ 


$$
\begin{gathered}
T R W B_{m l}=\sum_{i} \sum_{k \neq m} \sum_{j \neq m} r_{i k l j} w_{i j} x_{i k m j} \\
x_{i k m j}, z_{i k}, u_{k l} \in\{1,0\} \quad \forall i, k, m, l, j \\
T R W_{k}, T R R_{m}, T R W B_{k l}, T R W B_{m l} \geq 0 \quad \forall k, m, l
\end{gathered}
$$

Constraint (6) specifies the limitation on the number of hubs. Constraint (7) ensures that each non-hub node can be allocated to utmost one hub node. Constraints (8) and (9) imply that the entire flow shall pass through hubs, preventing the flow from being sent to non-hub nodes. Constraint (10) expresses that the failed node is a hub node and it may have only one backup hub. Constraint (11) indicates that the backup node is a hub node other than the one at which failed hub is deployed. Constraints (12) and (13) were explained previously. Constraints (14) and (15) set the nature of the problem variables.

\subsection{Linearization of the model}

Given that the objective function contains multiplications between discrete binary variables and continuous ones, we need to linearize the problem before we can solve it using linear programming. In the problem under study, we are dealing with the binary variables $u$ and $z$ and continuous variables $T R W B_{k l}$ and $T R W B_{m l}$ exist. On this basis, we consider two continuous variables $\lambda_{k l}$ and $\beta_{m l}$ with their definitions given as Eqs. (16) and (17), respectively:

$$
\begin{aligned}
& \lambda_{k l}=T R W B_{k l} u_{k l} \\
& \beta_{m l}=T R W B_{m l} u_{m l}
\end{aligned}
$$

Following with the research, the problem was linearized by adding to it the following constraints (where the parameter $v$ is a very large number).

$$
\begin{gathered}
\lambda_{k l} \leq v_{1} u_{k l} \quad \forall k, l \\
\lambda_{k l} \leq T R W B_{k l} \quad \forall k, l \\
\lambda_{k, l} \geq T R W B_{k l}-v_{1}\left(1-u_{k l}\right) \quad \forall k, l \\
\beta_{m l} \leq v_{2} u_{m l} \quad \forall m, l \\
\beta_{m l} \leq T R W B_{m l} \quad \forall m, l \\
\beta_{m l} \geq T R W B_{m l}-v_{2}\left(1-u_{m l}\right) \quad \forall m, l \\
\beta_{m l}, \lambda_{k l}, \theta_{k l} \geq 0 \forall m, l, k
\end{gathered}
$$

Thus, objective functions of the model can be rewritten as follows:

$$
\max o b j 1=\sum_{i} \sum_{k} \sum_{m} \sum_{j} r_{i k m j} x_{i k m j} w_{i j}
$$

$$
\begin{aligned}
& \max o b j_{2}=\sum_{i} \sum_{k} \sum_{m} \sum_{j} r_{i k m j} x_{i k m j} w_{i j} \\
& +\sum_{k} \sum_{l} \lambda_{k l} q_{k} \\
& +\sum_{m} \sum_{l}^{l} \beta_{m l} q_{m}
\end{aligned}
$$

Various methods have been proposed for solving multiobjective problems, such as weighting factors, epsilonconstraint ( $\varepsilon$-Constraint), etc. But these methods are not appropriate for the objective functions where the objectives exhibit dependencies upon or priorities over one another. In such cases, one can use so-called lexicographic method, which is thoroughly explained in the following.

The Fishburn's lexicography method [41] begins with prioritizing the objective functions based on their importance, followed by considering each of the objective functions as the main objective function of the problem in the order of priority. Suppose that the set $\left\{i=i_{1} \ldots i_{k}\right\}$ denotes prioritized objective functions; then the most important objective function $\left(i_{1}\right)$ is optimized in the feasible space $\delta$, as indicated in the following.

$$
\begin{aligned}
& \min f_{i 1}(x) \\
& \text { s.t } x \in \delta
\end{aligned}
$$

If the solution of the first objective function was not optimal in terms of the second objective function, then we proceed to the next objective function and select it as the main objective function.

$$
\begin{aligned}
& \min f_{i 2}(x) \\
& \text { s.t } f_{i_{1}} \leq c_{i_{1}}^{*} \quad x \in \delta
\end{aligned}
$$

$c_{i 1}^{*}$ is the optimum value of the first objective function in the problem described by Eq. (27). In other words, the second objective function is optimized as long as its value is worse than a predefined desired value. If the solution of Eq. (28) turns out to be sub-optimal, the third objective function is optimized as the next priority; this flow continues until an optimal solution is found. One of the challenges faced when using the lexicographic method is the large number of the operations to be performed to reach an optimum solution. As a workaround, some lower and upper bounds are imposed into the problem depending on the type of the objective function(s).

The considered problem in this research had two objective functions which depend on one another. As such, the lexicographic approach could be used for solving the problem. In this method, we begin with taking the first objective function into account and solving the problem independently. Then, when it comes to the second objective function, the solution of the first objective function remains unchanged and rather added to the model as a lower bound (i.e., a new constraint).

$$
\max o b j 1=\sum_{i} \sum_{k} \sum_{m} \sum_{j} r_{i k m j} x_{i k m j} w_{i j}
$$




$$
\begin{aligned}
& \max o b j_{2}=\sum_{i} \sum_{k} \sum_{m} \sum_{j} r_{i k m j} x_{i k m j} w_{i j} \\
& +\sum_{k} \sum_{l} \lambda_{k l} q_{k} \\
& +\sum_{m} \sum_{l} \beta_{m l} q_{m} \\
& \sum_{i} \sum_{k} \sum_{m} \sum_{j} r_{i k m j} x_{i k m j} w_{i j}=f_{1}^{*}
\end{aligned}
$$

Other constraints are incorporated into the model with no limitation. The only change would be the addition of the new constraint as the lower bound of the problem, where $f_{1}^{*}$ denotes the optimum objective function of the problem.

\section{Problem-solving algorithm}

The memetic algorithm is a combination of genetic algorithm with nearest-neighbor search (NNS). The purpose of the memetic algorithm is to evolve an initial population of solutions with the greatest diversity in search for an optimum solution. Since individual meta-heuristic algorithms might end up trapped at local optima, a hybrid metaheuristic algorithm (memetic) was used in this research.

The aim of searching is generally to meet two goals, namely exploring and exploiting the problem space. By exploring we mean to ensure the comprehensiveness of the search, and it is important since the problem space shall be searched thoroughly for finding the universal optimum solution. When it comes to exploiting, we are searching for a better solution around an obtained solution, i.e., local search. Genetic algorithms enjoy robust structure for a comprehensive search, but when it comes to the subjectmatter of exploiting, they need to be combined with some local search algorithm. So, selection of a local search method is important, because a particular local search algorithm may be suitable for a particular problem but rather fail to perform adequately in another problem. The memetic algorithm is a combination of the conventional genetic algorithm with some local search algorithm. The incorporation of the local search algorithm enhances the efficiency of searching. In the present research, a combination of genetic algorithm with simulated annealing algorithm was used to formulate a memetic algorithm. Simulated annealing algorithm was adopted to improve the solutions and increase the convergence rate.

The algorithm was performed through the following steps:

1) Start

2) Generate an initial population

3) Calculate fitness function for the first objective function

4) Perform crossover operation on the population and calculate fitness value

5) Perform mutation operation and calculate fitness value
6) Add up the initial population and the ones obtained after crossover and mutation operations

7) Select a random member of the resulting population and develop a neighborhood around it

8) If a better solution was found in the neighborhood, then go to the next step; otherwise calculate the probability of selecting a worse solution, and if there was a worse solution, then go to the next step; otherwise go to step 7.

9) Replace the selected member with the solution found in the neighborhood

10) Investigate the stopping criterion for simulated annealing algorithm; if stopping criterion holds, then go to the next step; otherwise go back to step 7.

11) If stopping criterion for the first objective function holds, then go to the next step; otherwise select the next generation and go to step 2 to repeat the steps from the beginning.

12) Generate an initial population for the second objective function

13) Select a member of the population at random and develop a neighborhood around it

14) If the solution found within the neighborhood is better, go to the next step; otherwise calculate the probability of selecting a worse solution, and if there is a worse solution, then go to the next step; otherwise go to the step 13.

15) Replace the selected member with the solution found within the neighborhood

16) Investigate stopping criterion for the second objective function; if it holds, terminate the algorithm; otherwise go to step 12 .

As it is observed from the algorithm steps, the algorithm begins with generating an initial population which is then subjected to crossover and mutation operations. In the next step, a new generation is selected and if the stopping criterion holds, the first objective function is calculated and the problem uses simulated annealing algorithm to solve the second objective function. But if the stopping criterion does not hold and the problem is trapped in a local optimum, then the algorithm uses simulated annealing algorithm to perform a better search of the solution space. Once finished with finding the optimum solution for the first objective function, simulated annealing algorithm is used to solve the problem for the second objective function as follows.

In order to solve an optimization problem, simulated annealing algorithm begins with an initial solution and then proceeds toward other solutions within a neighborhood around the solution following an iterative cycle. In case any of the solutions found in the neighborhood were superior over the current solution, the algorithm replaces the current solution with the solution found in the neighborhood (proceeds towards that).

Otherwise, the algorithm admits the solution as the current best solution with the probability of exp $(-\Delta E / T)$ 
(where $\Delta E$ is the difference between the values of an objective function for the present solution and that found within the neighborhood, and $T$ is a parameter called temperature). At each temperature, several iterations are undertaken before the temperature is reduced slowly. In early steps, the temperature is set to a very high value to enhance the probability of accepting worse solutions. However, as the temperature is decreased slowly, there will be less probability for worse solutions to be accepted, i.e., the algorithm converges toward a good solution. Simulated annealing algorithm is an unconstrained algorithm that is commonly applied for difficult designs. In the present research, simulated annealing algorithm was used as a neighborhood search algorithm; combining this algorithm with a genetic algorithm, the hybrid memetic algorithm was achieved. The stopping criterion for this algorithm and simulated annealing algorithm for the second objective function of the problem was defined as the situation where either no better solution than the current solution can be found or further reduction of temperature is impossible.

Table 1. Generation of a random matrix.

\begin{tabular}{lllll}
\hline 0.6625 & 0.9575 & 0.9416 & 0.9575 & 0.6655 \\
0.6524 & 0.5426 & 0.1446 & 0.8176 & 0.4113 \\
0.8511 & 0.1786 & 0.0948 & 0.3257 & 0.3659 \\
0.7508 & 0.2546 & 0.3847 & 0.0957 & 0.4182 \\
0.8746 & 0.9541 & 0.2043 & 0.0852 & 0.8044 \\
\hline
\end{tabular}

Table 2. Selection of hub nodes in the matrix.

\section{Algorithm-generated solution representation}

In order to better illustrate the chromosomes, we assume that the designed network consists of five nodes and three hubs. Accordingly, a dimensial $5 \times 5$ matrix filled with random values in the range of $[0,1]$ was considered. Now, in order to determine the three hubs across the network, three members with the largest values on the main diagonal of the matrix were selected as hubs, with the members with the largest values along each column selected as the nonhub nodes and assigned to the selected hub along the corresponding row. Then, the values of the elements along the rows with no hub nodes were set to zero. This process is described in table 1.

According to table 2, the three members with the largest values on the main diagonal were selected as hubs (green elements in the table).

As shown in table 3 , the elements on the rows with no hub nodes were set to zero (blue elements in the table).

Now, as shown in table 4, the element with maximum value along each column was selected as the non-hub node (marked in red in the table) and allocated to the corresponding hub node, as indicated in the last line of tables 4 and 5 .

In order to determine backup hubs, an array of length $p$ was used. Each element in this array had a value between 1 and $\mathrm{p}$. The $n$th element of the array determines the backup hub for the $n$th hub.

As it is evident, the nodes 1,2 , and 5 were selected as the hub nodes. When it came to representation, number 2

\begin{tabular}{|c|c|c|c|c|}
\hline hub & 0.9575 & 0.9416 & 0.9575 & 0.6625 \\
\hline 0.4113 & hub & 0.1446 & 0.5426 & 0.6524 \\
\hline 0.3659 & 0.3257 & 0.0948 & 0.1786 & 0.8511 \\
\hline 0.4182 & 0.0957 & 0.3847 & 0.2546 & 0.7508 \\
\hline 0.8044 & 0.0852 & 0.2043 & 0.9541 & hub \\
\hline
\end{tabular}

Table 3. Setting to zero the rows without hub nodes.

\begin{tabular}{|c|c|c|c|c|}
\hline hub & 0.9575 & 0.9416 & 0.9575 & 0.6625 \\
\hline 0.4113 & hub & 0.1446 & 0.5426 & 0.6524 \\
\hline 0 & 0 & 0 & 0 & 0 \\
\hline 0 & 0 & 0 & 0 & 0 \\
\hline 0.8044 & 0.0852 & 0.2043 & 0.9541 & hub \\
\hline
\end{tabular}


Table 4. Allocation of non-hub nodes to hub nodes.

\begin{tabular}{|c|c|c|c|c|}
\hline hub & 0.9575 & 0.9416 & 0.9575 & 0.6625 \\
\hline 0.4113 & hub & 0.1446 & 0.5426 & 0.6524 \\
\hline 0 & 0 & 0 & 0 & 0 \\
\hline 0 & 0 & 0 & 0 & 0 \\
\hline 0.8044 & 0.0852 & 0.2043 & 0.9541 & hub \\
\hline 1 & 2 & 1 & 1 & 5 \\
\hline
\end{tabular}

Table 5. Backup hub design.

\begin{tabular}{llll}
\hline Selected hubs & 1 & 2 & 5 \\
Representation of backup & 2 & 3 & 3 \\
$\quad$ hubs & & & \\
\hline
\end{tabular}

Table 6. Backup hub representation.

$\overline{2} 5$

implies that the second hub (hub 2) is selected as the backup hub. Further, number 3 implies that the third hub (hub 5) is selected as the backup hub. Tables 6 and 7 presents backup hubs.

Since backup hubs are selected from a pool of selected hubs, this is a good approach to representation. This representation is possible because backup hubs are selected independently.

In order to further elaborate on this representation, consider a hub network based on 10 nodes.

As it is observed in figure 2, the nodes 2, 5, and 3 are selected as hubs. Now, one should proceed to select backup hubs. Solution representation matrix is filled as (3-3-1); that is, the first hub (hub 2) and the third hub (hub 3) are selected as backup hubs, with the backup hub represented as (2-3-3). The initial population of the problem was generated as a random matrix whose elements ranged within

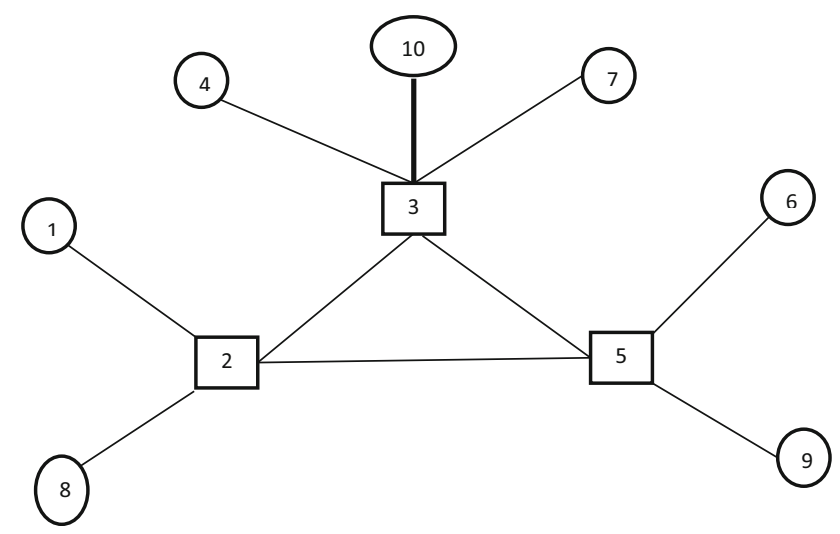

Figure 2. An example of the designed hub network.

$[0,1]$, with the number of rows and columns being equal to the number of nodes in the considered problem.

\subsection{Procedure for applying crossover operator}

Continuous uniform crossover operation was practiced in the present research. For this purpose, firstly, two parents were selected using roulette wheel method; then a continuous random mask of a size equal to chromosome length was generated, and each offspring got heritage from each parent according to this random mask: $y=\alpha * x_{1}+(1-\alpha) * x_{2}$

Table 7. Crossover operator

\begin{tabular}{|c|c|c|c|c|c|}
\hline Parent 1 & 0.49 & 0.62 & 0.37 & 0.84 & 0.08 \\
\hline Random mask & 0.24 & 0.31 & 0.54 & 0.48 & 0.95 \\
\hline Parent 2 & 0.80 & 0.47 & 0.16 & 0.46 & 0.91 \\
\hline Offspring 1 & 0.72 & 0.52 & 0.28 & 0.65 & 0.83 \\
\hline Offspring 2 & 0.59 & 0.58 & 0.26 & 0.66 & 0.16 \\
\hline
\end{tabular}


From the equation above for calculating first gene of offspring 1 , we have $\mathrm{y}=0.24 \times 0.49+(1-$ $0.24) \times 0.80=0.72$.

\subsection{Procedure for mutation operation}

In this case, the initial population size is multiplied by a mutation rate. For example, suppose that initial population is 10 in size and the mutation rate is 0.3 . This implies that 3 of the above cells shall be selected randomly and added to/subtracted from a random number.

\subsection{Fitness function}

Fitness function of the problem was the same as the objective function of the problem. Once finished with generating the initial population and selecting the superior parents, based on the selection function, for crossover operation, the mutation operator was applied and fitness function of the algorithm opted for the highest possible value as the objective function value; this process was continued until the best solution was found. Not to say that, this procedure was followed for the first objective function of the algorithm, while the second objective function followed principles of the simulated annealing algorithm.

\section{Computational results}

\subsection{Computational results obtained from US Airlines data}

In this section, $\mathrm{CAB}$ data for problems with 10,15 , and 25 nodes were used to validate the model. Meanwhile, CPLEX solver in GAMS Software and the designed metaheuristic algorithm, which was coded in Matlab, were used to solve the model. Three values of reliability parameter were used for the analysis, namely $0.2,0.4$, and 0.8 . The number of hubs in the problems with 10,15 , and 25 nodes were (3), (3, $5)$, and $(3,5,7)$, respectively. Given that GAMS Software was unable to handle problems with more than 10 nodes, it was utilized to analyze the 10-node problem only, with the other problems handled using the designed algorithm.

Furthermore, in all the problems, population size, the number of iterations, crossover rate, and mutation rate were set to $50,100,0.8$, and 0.3 , respectively. Furthermore, initial temperature and annealing rate were set to 10 and 0.1 , respectively. Given that lexicographic method suits small-scale problems (as mentioned in a previous section), the first objective function was taken as the most important objective function.

It should be noted that, given that it was extremely difficult to access reliability at each node across the network, based on O'Kelly, we used as reliability parameter a symmetric matrix whose elements ranged within $[0,1]$ and its diagonal

Table 8. Flow statistics for $\mathrm{CAB}$ data with 10 nodes.

\begin{tabular}{rrrrrrrrrrr}
\hline & 1 & 2 & \multicolumn{1}{c}{3} & \multicolumn{1}{c}{4} & 5 & 6 & 7 & 8 & 9 \\
\hline 1 & 0 & 6469 & 7629 & 20036 & 4690 & 6194 & 11688 & 2243 & 8857 & 7248 \\
2 & 6469 & 0 & 12999 & 13692 & 3322 & 5576 & 3878 & 3202 & 6699 & 4198 \\
3 & 7629 & 12999 & 0 & 35135 & 5956 & 14121 & 5951 & 5768 & 16578 & 4242 \\
4 & 20036 & 13692 & 35135 & 0 & 19094 & 35119 & 21423 & 27342 & 51341 & 15826 \\
5 & 4690 & 3322 & 5956 & 19094 & 0 & 7284 & 3102 & 1562 & 7180 & 1917 \\
6 & 6194 & 5576 & 14121 & 35119 & 7284 & 0 & 5023 & 3512 & 10419 & 3543 \\
7 & 11688 & 3878 & 5951 & 21423 & 3102 & 5023 & 0 & 11557 & 6479 & 34261 \\
8 & 2243 & 3202 & 5768 & 27342 & 1562 & 3512 & 11557 & 0 & 5615 & 7095 \\
9 & 8857 & 6699 & 16578 & 51341 & 7180 & 10419 & 6479 & 5615 & 0 \\
10 & 7248 & 4198 & 4242 & 15826 & 1917 & 3543 & 34261 & 7095 & 4448 & 0 \\
\hline
\end{tabular}

Table 9. Reliability of the 10 nodes across the network.

\begin{tabular}{lllllllllllcc}
\hline & 1 & 2 & 3 & 4 & 5 & 6 & 7 & 8 & 9 \\
\hline 1 & 1 & 0.95 & 0.73 & 0.46 & 0.53 & 0.27 & 0.34 & 0.83 & 0.2 & 0.66 \\
2 & 0.95 & 1 & 0.77 & 0.5 & 0.65 & 0.26 & 0.39 & 0.87 & 0.17 & 0.82 \\
3 & 0.73 & 0.77 & 1 & 0.67 & 0.52 & 0.38 & 0.61 & 0.66 & 0.28 & 0.88 \\
4 & 0.46 & 0.5 & 0.67 & 1 & 0.26 & 0.3 & 0.58 & 0.38 & 0.02 & 0.6 \\
5 & 0.53 & 0.65 & 0.52 & 0.26 & 1 & 0.71 & 0.37 & 0.64 & 0.79 & 0.68 \\
6 & 0.27 & 0.26 & 0.38 & 0.3 & 0.71 & 1 & 0.76 & 0.38 & 0.55 & 0.55 \\
7 & 0.34 & 0.39 & 0.61 & 0.58 & 0.37 & 0.76 & 1 & 0.25 & 0.27 & 0.55 \\
8 & 0.83 & 0.87 & 0.66 & 0.38 & 0.64 & 0.38 & 0.25 & 1 & 0.31 \\
9 & 0.2 & 0.17 & 0.28 & 0.02 & 0.79 & 0.55 & 0.27 & 0.31 & 1 & 0.77 \\
10 & 0.66 & 0.82 & 0.88 & 0.6 & 0.68 & 0.55 & 0.55 & 0.77 & 0.39 & 0.39 \\
\hline
\end{tabular}


elements were equal to 1 . Also, the probability of hub failure, $q$, was indicated by a number within the range of $[0.1,0.3]$. The rate of dispatched flow and reliability for the case with 10 nodes are reported in Tables 8 and 9. It should be noted that, all of the problem results were obtained by a PC equipped with 4 GB of RAM and a Core i7 CPU.

According to table 10, the meta-heuristic algorithm designed for small problems had no significant gap (distance from optimum solution) for the first objective function, although it returned a negligible gap for the second objective function. The gap can be evaluated from the following equation:

$$
\mathrm{Gap}=\frac{\text { best sol of the algorithm }- \text { best sol of games }}{\text { best sol of the algorithm }}
$$

As observed in table 11, six sample problems were designed for the case with 15 nodes, with the results reported for 3 and 5 hubs. Solutions of the problems are reported for different values of reliability, namely $0.2,0.4$, and 0.8 . It should be noted that GAMS Software was unable to handle the problem with 15 nodes.

As seen on figure 3, in all of the above cases with 15 nodes, the value of the objective function was higher when backup hubs were considered, rather than the case with no backup hub.

This indicates accuracy of the model because this model could pass maximum possible flow through the network in the second case.

As observed in table 12, we further studied the problem on a case with 25 nodes and either 3, 5, or 7 hubs at three levels of reliability, namely $0.2,0.4,0.8$.

Since the reliability factor between a pair of hubs facilitates the flow between the two hubs and given that the reliability factor of a route is calculated as $r(i, k) * r(k, m)^{(1-\alpha)} * r(m, j)$ where reliability factor appears as an exponent, the higher the reliability factor, the higher would be the flow capacity through the network. In addition, reliability factor tends to affect the choice of selected hubs and backup hubs; taking a look at the highlighted section of figure 4, one may found how a change in the selected hubs changes backup hubs. An important point to note is the direct effect of the reliability factor on not only the network reliability and hence the dispatched flow, but also on the choice of backup hubs. As it is observed in figure 4 , by increasing the reliability factor, one could transfer a great deal of flow through the network. In table 2, the first objective function of the problem is studied for three values of reliability factor, namely $0.2,0.6$, and 0.8 .

\subsection{Computational results obtained from Turkish network data}

In this section, the algorithm is further validated based on data from Turkish Airline Network with 81 nodes.

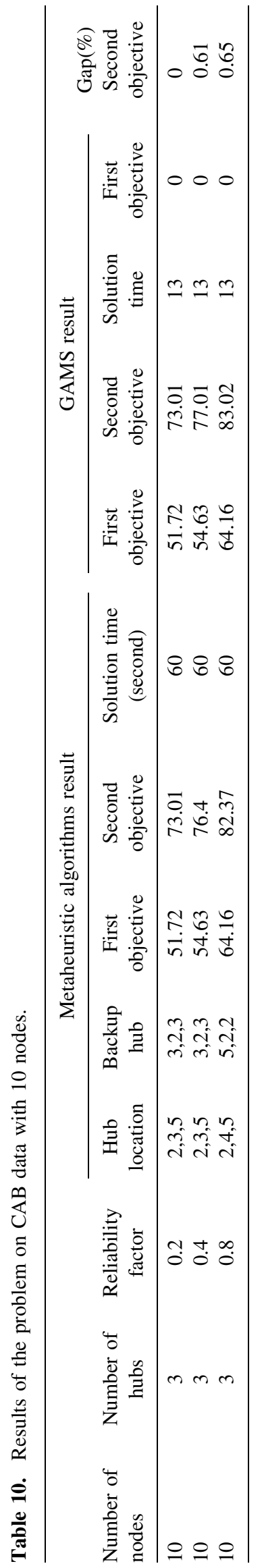


Table 11. Results of 15-node problem derived from CAB data.

\begin{tabular}{|c|c|c|c|c|c|c|c|}
\hline \multirow[b]{2}{*}{ Number of nodes } & \multirow[b]{2}{*}{ Number of hubs } & \multirow[b]{2}{*}{$\begin{array}{l}\text { Reliability } \\
\text { factor }\end{array}$} & \multicolumn{4}{|c|}{ Metaheuristic algorithm result } & \multirow[b]{2}{*}{$\begin{array}{l}\text { Solution } \\
\text { time }\end{array}$} \\
\hline & & & $\begin{array}{c}\text { Hub } \\
\text { location }\end{array}$ & Backup hub & $\begin{array}{c}\text { First } \\
\text { objective }\end{array}$ & $\begin{array}{c}\text { Second } \\
\text { objective }\end{array}$ & \\
\hline 15 & 3 & 0.2 & $2,12,15$ & $12,2,12$ & 125.30 & 173.15 & 22 \\
\hline 15 & 3 & 0.4 & $3,4,15$ & $4,3,3$ & 131.78 & 177.13 & 22 \\
\hline 15 & 3 & 0.8 & $3,4,14$ & $4,3,3$ & 152.91 & 207.11 & 22 \\
\hline 15 & 5 & 0.2 & $2,5,11,12,15$ & $12,15,12,2,5$ & 131.48 & 192.49 & 25 \\
\hline 15 & 5 & 0.4 & $2,3,4,11,15$ & $3,11,3,3,3$ & 142.78 & 201.95 & 25 \\
\hline 15 & 5 & 0.8 & $2,3,4,7,14$ & $3,2,3,3,7$ & 174.453 & 242.13 & 25 \\
\hline
\end{tabular}

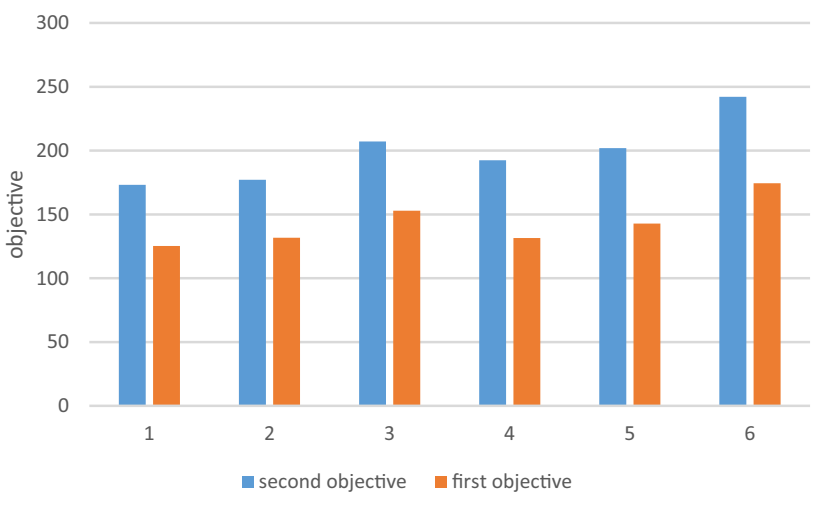

Figure 3. Comparison between objectives.

Table 13 reports results of the problem designed based on the 81-node Turkish Airline Network considering 3 and 5 hubs. As is observed, optimal solution was found within a reasonable time for such a large problem.

Figure 4 shows higher values for the second objective function, as compared to the first one, at different iterations, indicating good performance of the designed model when applied to Turkish Airline Network data, as indicated by increased flow across the network by considering backup hubs. Meanwhile, the algorithm succeeded to solve the problem within a reasonable deal of time, further confirming appropriate performance of the problem in terms of processing time.

Our computational results indicate that designing a network without considering reliability issues or backup hub(s) ends up losing some flow through the network. For example, when the set of hubs $(33,65,66)$ were selected in the network, the hubs $(65,33,65)$ served as backup hubs, and should one is willing to calculate the lost flow following this approach when the backup hub is not in service, he/she can simply subtract the value of the second objective function (first row of table 13) from the value of the first objective function.

This difference is well evident in figure 5 , where the second objective function dispatches more flow than that of the first objective function. As such, it is found that some $18 \%$ of our flow is missed as hubs go out of service. Thus, the proposed mathematical model is well capable of exploiting maximum network capacity for transferring the flow.

\subsection{Computational results obtained from Iranian Aviation Data (IAD)}

Figure 6 shows all of the points under study across the map of Iran. This figure shows the distribution of 37 Iranian cities hosting airports. In this example, the aim is to test the

Table 12. Results of 25-node problem based on CAB data.

\begin{tabular}{|c|c|c|c|c|c|c|c|}
\hline \multirow[b]{2}{*}{$\begin{array}{l}\text { Number of } \\
\text { nodes }\end{array}$} & \multirow[b]{2}{*}{$\begin{array}{c}\text { Number of } \\
\text { hubs }\end{array}$} & \multirow[b]{2}{*}{$\begin{array}{l}\text { Reliability } \\
\text { factor }\end{array}$} & \multicolumn{4}{|c|}{ Metaheuristic algorithm result } & \multirow[b]{2}{*}{$\begin{array}{l}\text { Solution } \\
\text { time }\end{array}$} \\
\hline & & & Hub location & Backup hub & $\begin{array}{c}\text { First } \\
\text { objective }\end{array}$ & $\begin{array}{c}\text { Second } \\
\text { objective }\end{array}$ & \\
\hline 25 & 3 & 0.2 & $10,12,20$ & $12,10,12$ & 510.37 & 742.21 & 43 \\
\hline 25 & 3 & 0.4 & $10,12,20$ & $12,10,12$ & 524.04 & 759.41 & 43 \\
\hline 25 & 3 & 0.8 & $3,17,20$ & $20,3,3$ & 561.14 & 796.57 & 45 \\
\hline 25 & 5 & 0.2 & $2,3,5,12,20$ & $3,12,12,3,12$ & 527.68 & 802.7 & 48 \\
\hline 25 & 5 & 0.4 & $3,10,12,15,17$ & $12,15,3,3,3$ & 539.47 & 790.46 & 48 \\
\hline 25 & 5 & 0.8 & $3,7,14,17,22$ & $22,3,3,3,3,3$ & 654.5 & 905.94 & 48 \\
\hline 25 & 7 & 0.2 & $2,3,5,10,12,20,25$ & $10,10,25,3,3,12,20$ & 534.43 & 809.37 & 40 \\
\hline 25 & 7 & 0.4 & $3,5,15,17,20,22,25$ & $17,15,17,20,22,25$ & 557.16 & 787.44 & 40 \\
\hline 25 & 7 & 0.8 & $3,4,6,12,14,17,20$ & $17,12,17,3,17,3,12$ & 675.22 & 947.98 & 40 \\
\hline
\end{tabular}


Table 13. Results of the proposed meta-heuristic algorithm on 81 nodes across Turkish Airline Network.

\begin{tabular}{|c|c|c|c|c|c|c|c|}
\hline \multirow[b]{2}{*}{$\begin{array}{l}\text { Number of } \\
\text { nodes }\end{array}$} & \multirow[b]{2}{*}{$\begin{array}{c}\text { Number of } \\
\text { hubs }\end{array}$} & \multirow[b]{2}{*}{$\begin{array}{l}\text { Reliability } \\
\text { factor }\end{array}$} & \multicolumn{4}{|c|}{ Metaheuristic algorithm result } & \multirow[b]{2}{*}{$\begin{array}{l}\text { Solution } \\
\text { time }\end{array}$} \\
\hline & & & Hub location & Backup hub & $\begin{array}{c}\text { First } \\
\text { objective }\end{array}$ & $\begin{array}{c}\text { Second } \\
\text { objective }\end{array}$ & \\
\hline 81 & 3 & 0.2 & $33,65,66$ & $65,33,65$ & 3822.25 & 4662.39 & 300 \\
\hline 81 & 3 & 0.4 & $30,37,42$ & $37,42,37$ & 4462.34 & 5703.87 & 300 \\
\hline 81 & 3 & 0.8 & $53,55,67$ & $67,67,55$ & 4142.05 & 5622.86 & 300 \\
\hline 81 & 5 & 0.2 & $37,42,60,67,72$ & $67,37,37,42,67$ & 4191.45 & 5411.69 & 310 \\
\hline 81 & 5 & 0.4 & $27,33,34,36,47$ & $47,36,47,47,34$ & 4307.4 & 5797.42 & 310 \\
\hline 81 & 5 & 0.8 & $19,34,48,50,58$ & $48,50,50,48,34$ & 4722.74 & 6530.75 & 310 \\
\hline
\end{tabular}

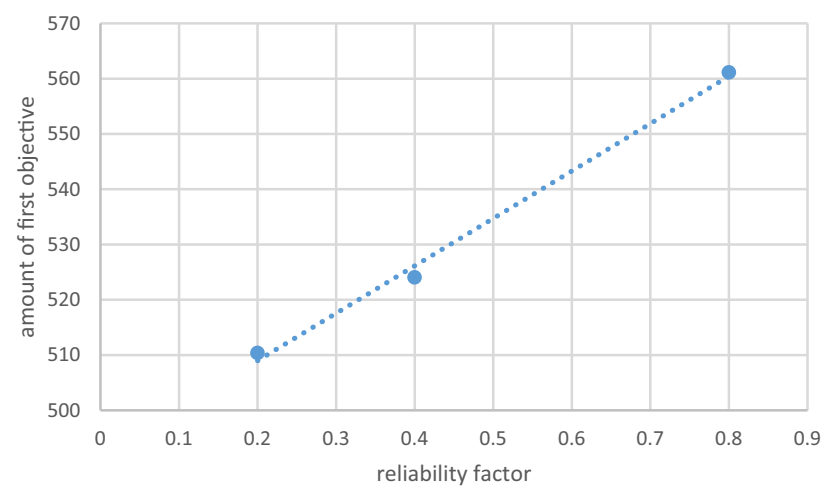

Figure 4. The increase value of the objective function by increasing the reliability factor.

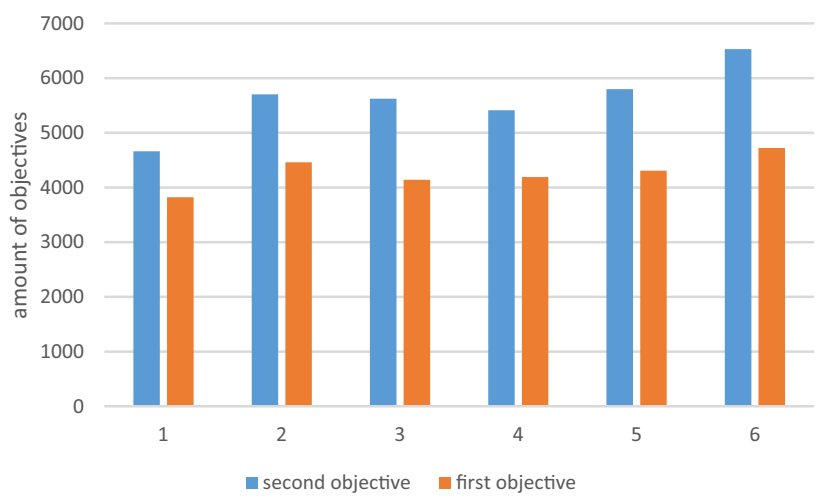

Figure 5. A comparison between values of the objective based on data from Turkish Airline Network.

application of the proposed model on the data obtained from Iranian aviation data (IAD), with the results given in table 14.

The first row of the table (shaded) indicates that the nodes $(19,31,36)$ were selected as hubs; these nodes correspond to Mashhad, Tehran and Zahedan, respectively, i.e., the choice of these cities as hubs was actually very reasonable since most of the Iranian air traffic actually passes through these cities. In another approach, when five nodes were to be selected as hubs, Ramsar, Shiraz, Tabriz, Tehran, and Zahedan cities were chosen as hubs, further confirming the model accuracy. Another remarkable point to analyze was the role of backup hubs used in the model, which turned out increasing the level of reliability and the value of the second objective function compared to the first objective function.

\subsection{Validation of the proposed algorithm}

In order to study the values of the algorithm parameters, the obtained algorithm was tested for the first objective function on US airlines data. For this purpose, the first objective function was considered alone to validate the algorithm, because GAMS Software failed to handle problems with more than 10 nodes. As such, a comparison was made on the values of the first objective function between GAMS Software and the designed algorithm.

As observed in table 15, the proposed problem was solved with the proposed meta-heuristic algorithm and gave zero gaps compared to the optimal solution of GAMS Software for problems of any size. Thus, it could be concluded that the proposed algorithm converged to the optimum solution within a reasonable deal of time

\subsection{Investigation of the algorithm convergence}

In order to study the rate of convergence achieved by the proposed algorithm, investigations were performed on 25 -node $\mathrm{CAB}$ data. A maximum number of iterations in each generation was considered as the stopping criterion for achieving the final solution. The criterion was further used to evaluate the algorithm convergence rate. This problem was used for $\mathrm{p}=3$ and reliability factor $=0.8$.

As observed in figure 7, from the 49th iteration on, the algorithm continued to return almost the same solution, confirming suitable convergence rate of the algorithm as it achieved an optimal solution within an appropriate number of iterations. 


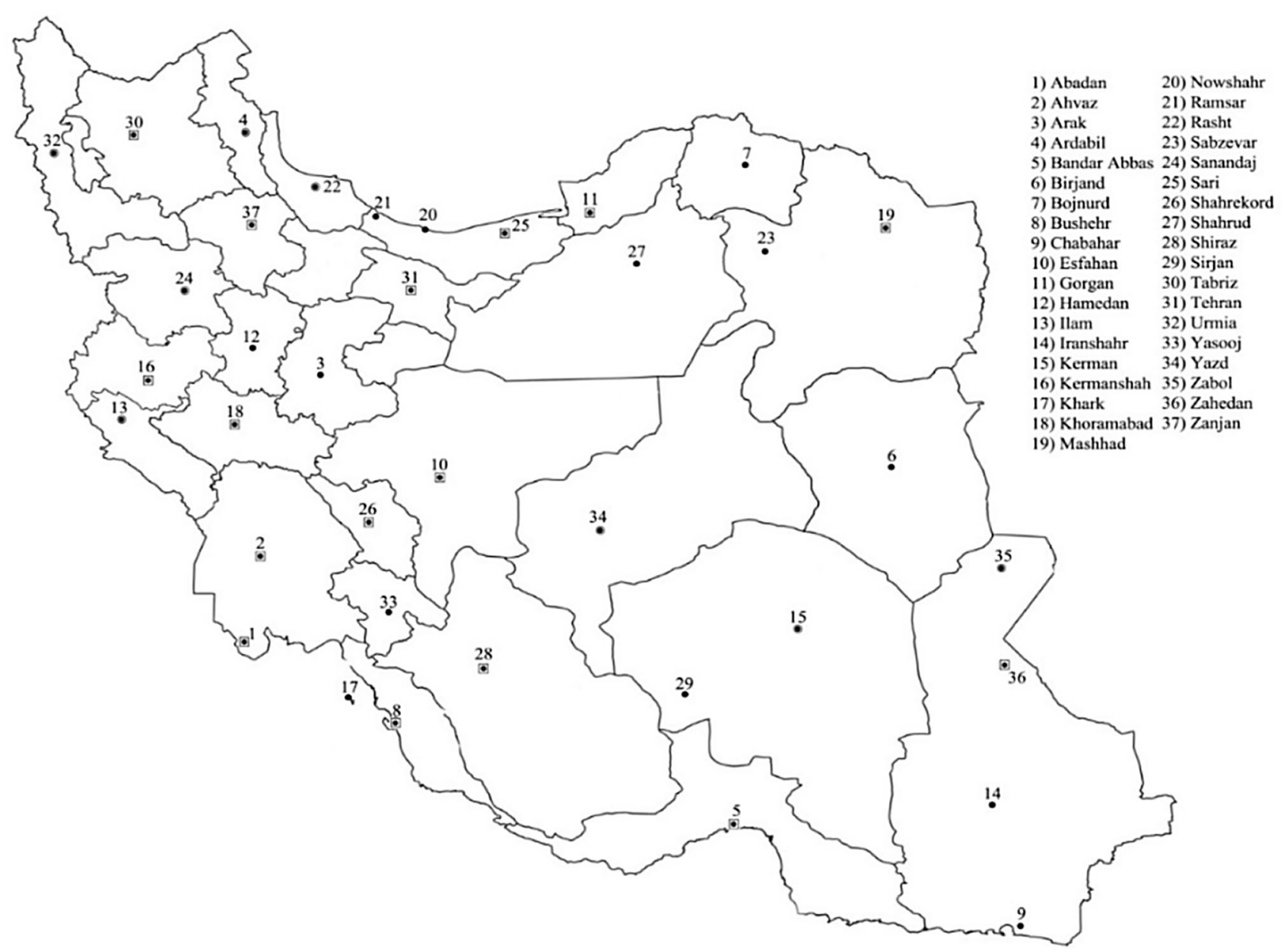

Figure 6. Map of Iran in terms of Iranian Airports data.

Table 14. Results of the proposed meta-heuristic algorithm on 37 nodes of Iranian aviation data.

\begin{tabular}{|c|c|c|c|c|c|c|c|}
\hline \multirow{2}{*}{$\begin{array}{l}\text { Number } \\
\text { of nodes }\end{array}$} & \multirow{2}{*}{$\begin{array}{l}\text { Number } \\
\text { of hubs }\end{array}$} & \multirow{2}{*}{$\begin{array}{l}\text { Reliability } \\
\text { factor }\end{array}$} & \multicolumn{4}{|c|}{ Metaheuristic algorithm result } & \multirow{2}{*}{$\begin{array}{l}\text { Solution } \\
\text { time }\end{array}$} \\
\hline & & & Hub location & Backup hub & $\begin{array}{c}\text { First } \\
\text { objective }\end{array}$ & $\begin{array}{c}\text { Second } \\
\text { objective }\end{array}$ & \\
\hline 37 & 3 & 0.2 & $19,31,36$ & $36,36,31$ & 77.61 & 99.48 & 76 \\
\hline 37 & 3 & 0.4 & $29,30,35$ & $35,35,29$ & 86.35 & 106.49 & 76 \\
\hline 37 & 3 & 0.8 & $30,36,37$ & $36,37,30$ & 90.29 & 114.25 & 76 \\
\hline 37 & 5 & 0.2 & $21,28,30,31,36$ & $30,36,31,36,31$ & 82.04 & 105.94 & 76 \\
\hline 37 & 5 & 0.4 & $15,30,31,36,37$ & $31,31,37,31,31$ & 90.06 & 115.63 & 78 \\
\hline 37 & 5 & 0.8 & $4,16,30,31,35$ & $30,31,31,30,31$ & 96.05 & 120.88 & 80 \\
\hline
\end{tabular}

\section{Conclusion}

Hub location problem is known to contribute to increased efficiency, manpower saving, and reduced transportation cost within communication networks. It is of paramount importance due to its numerous applications including those in air trips, post services, distribution systems, transportation industry, and computer networks. For these reasons, a bi-objective mathematical model was designed to consider the situations before and after a possible hub failure; the first objective was to pass maximum flow through the network considering route reliability, and the second objective was to prevent from wasting the flow as a result of a hub failure.

A mathematical multi-objective mixed-integer nonlinear programming model was designed for the problem. The model was then linearized into a linear multi-objective mixed-integer programming model to facilitate the solving 
Table 15. Validation of the proposed algorithm based on the value of the first objective function.

\begin{tabular}{|c|c|c|c|c|c|}
\hline Number of nodes & Number of hubs & Reliability factor & GAMS CPLEX solver & Metaheuristic algorithm & Gap \\
\hline 10 & 3 & 0.2 & 52.72 & 52.72 & 0 \\
\hline 10 & 3 & 0.4 & 54.63 & 54.63 & 0 \\
\hline 10 & 3 & 0.8 & 64.16 & 64.16 & 0 \\
\hline 10 & 4 & 0.2 & 52.67 & 52.67 & 0 \\
\hline 10 & 4 & 0.4 & 57.01 & 57.01 & 0 \\
\hline 10 & 4 & 0.8 & 70.93 & 70.93 & 0 \\
\hline 15 & 3 & 0.2 & 125.3 & 125.3 & 0 \\
\hline 15 & 3 & 0.4 & 131.78 & 131.78 & 0 \\
\hline 15 & 3 & 0.8 & 152.91 & 152.91 & 0 \\
\hline 15 & 4 & 0.2 & 128.76 & 128.76 & 0 \\
\hline 15 & 4 & 0.4 & 137.49 & 137.49 & 0 \\
\hline 15 & 4 & 0.8 & 165.47 & 165.47 & 0 \\
\hline 25 & 3 & 0.2 & 510.37 & 510.37 & 0 \\
\hline 25 & 3 & 0.4 & 524.04 & 524.04 & 0 \\
\hline 25 & 3 & 0.8 & 561.14 & 561.14 & 0 \\
\hline 25 & 4 & 0.2 & 512.68 & 512.68 & 0 \\
\hline 25 & 4 & 0.4 & 530.39 & 530.39 & 0 \\
\hline 25 & 4 & 0.8 & 634.51 & 634.51 & 0 \\
\hline
\end{tabular}

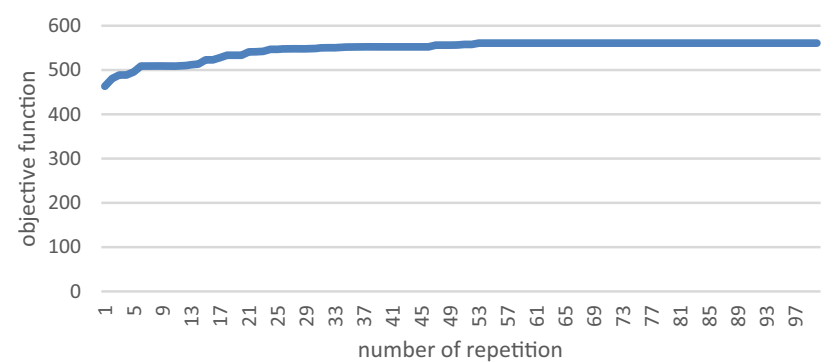

Figure 7. Convergence criterion of the algorithm in terms of the number of iterations.

stage. Given that the two objectives considered in the present research depended on one another, the lexicographic method was used to solve the problem when it was small in size. However, being NP-Hard, the problem is extremely difficult to solve when it gets large in size, so that a meta-heuristic algorithm called memetic algorithm (a combination of genetic and simulated annealing algorithms) was used to have it solved.

In order to show superior performance of the designed algorithm over GAMS optimization software, we used 30 examples developed based on Turkish Network, CAB, and IAD data, for all of which the proposed meta-heuristic algorithm outperformed GAMS Software in terms of processing time while returning solutions of comparable accuracy.

In this research, a preventive reliability approach was followed for the case with no hub failure considering route reliability, while backup hub approach was used for the case after a possible hub failure to prevent from wasting the flow as a result of the hub failure. The objective was to compare the two approaches to this problem. In fact, in the first approach, the objective function accepted the reliable routes only and tended to guide the flow toward the route of the highest reliability. That was while in the second approach, backup hubs were considered for the cases where primary hubs were out of service. Indeed the second approach is complementary to the first one because, in reality, there are cases where routes or hubs go out of service even by considering the highest levels of safety factors. As it was observed in the section on results, in all cases, the second objective function succeeded to handle greater levels of flow, as compared to that of the first objective function. This validated the proposed model whose main objective was to enhance network reliability for delivering more flows.

The following points are recommended for future research:

1) Considering backup hubs in multiple allocation problems, because there are cases where a non-hub node needs more than one hub to handle the flow.

2) Considering the probability of multiple hub failures at the same time.

3) Considering capacity limitations; here we considered the problem with no capacity limitation, but there may be conditions under which maximum capacity of the network is impossible to exploit.

4) Considering exact solution methods; given that hub location problem is categorized as a strategic problem, it is of paramount importance to design an exact solution method for this problem.

\section{References}

[1] Hekmatfar M and Pishvaee M 2009 Hub location problem. In Facility Location. Physica-Verlag Heidelberg, pp. 243-270 
[2] Chinchuluun A and Pardalos P M 2007 A survey of recent developments in multiobjective optimization. Ann. Oper. Res. 154(1): 29-50

[3] Krasnogor N and Smith J 2005 A tutorial for competent memetic algorithms: model, taxonomy and design issues. IEEE Tran. Evolut. Comput. 9(5): 474-488

[4] Rodríguez V, Alvarez M J and Barcos L 2007 Hub location under capacity constraints. Transp. Res. Part E. Logist. Transp. Rev. 43(5): 495-505

[5] Snyder L V and Daskin M S 2005 Reliability models for facility location: the expected failure cost case. Transp. Sci. 39(3): pp. 400-416

[6] Berman O, Krass D and Menezes M B 2007 Facility reliability issues in network p-median problems: strategic centralization and co-location effects. Oper. Res. 5(2): 332-350

[7] Zhan R L and Shen Z J M 2007 Models and algorithms for reliable facility location problems and system reliability optimization, Doctoral Dissertation, University of Florida

[8] Lim M, Daskin M S, Bassamboo A and Chopra S 2010 A facility reliability problem: Formulation, properties, and algorithm. Naval Res. Logist. 57(1): 58-70

[9] An Y, Zhang Y and Zeng B 2015 The reliable hub-and-spoke design problem: models and algorithms. Transp. Res. Part B Methodol. 77: 103-122

[10] Snyder L V 2006 Facility location under uncertainty: a review. IIE Trans. 38(7): 547-564

[11] Grubesic T H, O'Kelly M E and Murray A T 2003 A geographic perspective on commercial Internet survivability. Telemat. Inform. 20(1): 51-69

[12] Klincewicz J G 1998 Hub location in backbone/tributary network design: a review. Locat. Sci. 6(1-4): 307-335

[13] Skorin-Kapov D, Skorin-Kapov J and O'Kelly M 1996 Tight linear programming relaxation of uncapacitated p-hub median problems. Eur. J. Oper. Res. 94(3): 582-593

[14] O'Kelly M E 2009 Rectilinear minimax hub location problems. J. Geogr. Syst. 11(3): 227-241

[15] Parvaresh F, Husseini S M, Golpayegany S H and Karimi B 2014 Hub network design problem in the presence of disruptions. J. Intell. Manuf. 25(4): 755-774

[16] Hamidi M, Gholamian M and Shahanaghi K 2014 Developing prevention reliability in hub location models. Proc. Inst. Mech. Eng. Part O J. Risk Reliab. 228(4): 337-346

[17] Azizi N, Chauhan S, Salhi S and Vidyarthi N 2016 The impact of hub failure in hub-and-spoke networks: Mathematical formulations and solution techniques. Comput. Oper. Res. 65: 174-188

[18] Azizi N 2017 Managing facility disruption in hub-and-spoke networks: formulations and efficient solution methods. Ann. Oper. Res. 1-27. doi:https://doi.org/10.1007/s10479-0172517-0

[19] Eghbali M, Abedzadeh M and Setak M 2014 Multi-objective reliable hub covering location considering customer convenience using NSGA-II. Int. J. Syst. Assur. Eng. Manag. 5(3): 450-460

[20] Zarrinpoor N, Fallahnezhad M S and Pishvaee M S 2018 The design of a reliable and robust hierarchical health service network using an accelerated Benders decomposition algorithm. Eur J. Oper. Res. 265(3): 1013-1032

[21] Ghezavati V and Hosseinifar P 2018 Application of efficient metaheuristics to solve a new bi-objective optimization model for hub facility location problem considering value at risk criterion. Soft Comput. 22(1): 195-212

[22] Sohn J and Park S 1998 Efficient solution procedure and reduced size formulations for p-hub location problems. Eur. J. Oper. Res. 108(1): 118-126

[23] Contreras I, Díaz J A and Fernández E 2009 Lagrangean relaxation for the capacitated hub location problem with single assignment. OR Spectr. 31(3): 483-505

[24] O'Kelly ME 1987 A quadratic integer program for the location of interacting hub facilities. Eur. J. Oper. Res. 32(3): 393-404

[25] Acampora G, Loia V, Salerno S and Vitiello A 2012 A hybrid evolutionary approach for solving the ontology alignment problem. Int. J. Intell. Syst. 27(3): 189-216

[26] Galinier P, Boujbel Z and Fernandes M C 2011 An efficient memetic algorithm for the graph partitioning problem. Ann. Oper. Res. 191(1): 1-22

[27] Neri F, Cotta C and Moscato P (Eds). 2012 Handbook of memetic algorithms (Vol. 379). Heidelberg: Springer

[28] Chen J F 2007 A hybrid heuristic for the uncapacitated single allocation hub location problem. Omega 35(2): 211-220

[29] Pradeepmon T G and Paul B 2012 A simulated annealingbased hybrid algorithm for solving UFL problems. Int. J. Appl. Manag. Sci. 4(3): 274-283

[30] Saboury A, Ghaffari-Nasab N, Barzinpour F and Jabalameli M S 2013 Applying two efficient hybrid heuristics for hub location problem with fully interconnected backbone and access networks. Comput. Oper. Res. 40(10): 2493-2507

[31] Mokhtari N and Abbasi M 2015 Applying VNPSO algorithm to solve the many-to-many hub location-routing problem in a large scale. Eur. Online J. Natl Soc. Sci. Proc. 3(4): 647-656

[32] Amin-Naseri M R, Yazdekhasti A and Salmasnia A 2018 Robust bi-objective optimization of uncapacitated single allocation p-hub median problem using a hybrid heuristic algorithm. Neural Comput. Appl. 29(9): 511-532

[33] Zhalechian M, Torabi S A and Mohammadi M 2018 Huband-spoke network design under operational and disruption risks. Transp. Res. Part E Logist. Transp. Rev. 109: 20-43

[34] Mogale D G, Kumar S K and Tiwari M K 2016 Two-stage Indian food grain supply chain network transportation-allocation model. IFAC-PapersOnLine 49(12): 1767-1772

[35] Kartal Z, Hasgul and Ernst A T 2017 Single allocation p-hub median location and routing problem with simultaneous pick-up and delivery. Transp. Res. Part E Logist. Transp. Rev. 108: 141-159

[36] Bashiri M, Rezanezhad M, Tavakkoli-Moghaddam R and Hasanzadeh H 2018 Mathematical modeling for ap-mobile hub location problem in a dynamic environment by a genetic algorithm. Appl. Math. Modell. 54: 151-169

[37] Mogale D G, Kumar M, Kumar S K and Tiwari M K 2018 Grain silo location-allocation problem with dwell time for optimization of food grain supply chain network. Transp. Res. Part E Logist. Transp. Rev. 111: 40-69

[38] Mogale D G, Kumar S K and Tiwari M K 2018 An MINLP model to support the movement and storage decisions of the Indian food grain supply chain. Control Eng. Pract. 70: 98-113

[39] Maiyar L M and Thakkar J J 2017 A combined tactical and operational deterministic food grain transportation model: Particle swarm based optimization approach. Comput. Ind. Eng. 110: 30-42 
[40] Mogale D G, Kumar S K, Márquez F P G and Tiwari M K 2017 Bulk wheat transportation and storage problem of public distribution system. Comput. Ind. Eng. 104: 80-97
[41] Fishburn P C 1974 Exceptional paper-Lexicographic orders, utilities and decision rules: A survey. Manag. Sci. 20(11): 1442-1471 